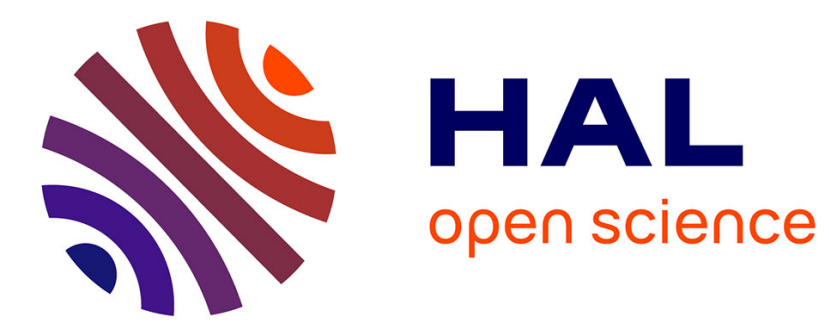

\title{
Le sifflement du serpent : du son inarticulé à la mise en musique
}

Sylvain Perrot

\section{To cite this version:}

Sylvain Perrot. Le sifflement du serpent: du son inarticulé à la mise en musique. S. Barbara, J. Trinquier. Ophiaka. Diffusion et réception des savoirs antiques sur les ophidiens, 47 (1), pp.345-361, 2012, Anthropozoologica. halshs-01632650

\section{HAL Id: halshs-01632650 https://shs.hal.science/halshs-01632650}

Submitted on 15 Oct 2019

HAL is a multi-disciplinary open access archive for the deposit and dissemination of scientific research documents, whether they are published or not. The documents may come from teaching and research institutions in France or abroad, or from public or private research centers.
L'archive ouverte pluridisciplinaire HAL, est destinée au dépôt et à la diffusion de documents scientifiques de niveau recherche, publiés ou non, émanant des établissements d'enseignement et de recherche français ou étrangers, des laboratoires publics ou privés. 


\title{
Le sifflement du serpent : du son inarticulé à la mise en musique
}

\author{
Sylvain PERROT \\ Université de Paris IV - Sorbonne / École Normale Supérieure - UMR AOROC \\ Sylvain.Perrot@ens.fr
}

MOTS CLÉS

serpent musique sifflement

aulos

Delphes pythique

KEY WORDS

Snake

Music

Hissing

Aulos

Delphi

Pythian

Perrot S. 2012. Le sifflement du serpent : du son inarticulé à la mise en musique. Anthropozoologica $47.1: 345-361$.

Dans la pensée grecque, les cris des animaux ne sauraient être associés au logos humain, car ils sont formés de sons inarticulés. Le serpent en est sans doute le paradigme : dans la plupart des cultures, son cri est généralement transcrit par une onomatopée si brève qu’elle ne convoque que la consonne sourde $[s]$, sans la moindre vocalisation. Au premier abord donc, on serait tenté de penser que le serpent est étranger à toute forme de musique : c'est la voyelle qui porte par excellence la mélodie, et les Grecs l'avaient bien compris dans leur système de notation musicale. Mais il faut dépasser cette première expérience acoustique. Le serpent est bel et bien considéré par les Grecs comme un animal musicien, au même titre que les cigales qui ont elles aussi un chant plutôt consonantique. Le paradoxe est évident quand on considère le nom grec donné au sifflement du serpent : syrigma. Si la racine porte bien l'onomatopée attendue, on constate que le même mot est employé pour désigner le son d'un instrument de musique, la célèbre flûte de Pan. Mieux encore, le sifflement de Python, qu'Apollon tue de ses flèches à Delphes, a donné lieu dès le $\mathrm{VI}^{\mathrm{e}}$ siècle av. J.-C. à un passage obligé de la composition musicale que tout artiste doit créer et interpréter dans les concours de Delphes, le nome pythique. En effet, dans une de ses parties, appelée syringes, le musicien devait reproduire avec l'aulos pythique, instrument spécifique aux concours delphiques, les sifflements du serpent agonisant sous les traits d'Apollon. L'enjeu est alors crucial : il s'agit de mettre en musique ce qui est proprement non-musical.

\section{ABSTRACT}

The hissing of the snake: how is it possible to set an inarticulate sound to music?

For a Greek mind animal cries cannot be associated to human logos, for being inarticulate sounds. One of the best examples is the snake, whose cry is usually transcribed by the consonant [s], without any vocalizing. Therefore, snakes seem not to be musicians: melody is produced by vocals, according to Greek treatises on music. However, snakes are considered as musician animals by Greeks, like cicadas. The word syrigma, that Greeks used to define the hissing of the snake, means also the sound of the Pan flute. Moreover, Pytho's hissing becomes a theme of a musical composition that every musician has to make and to perform at the Pythian Games, the nomos pythikos: in one of its parts, he has to depict, with his Pythian aulos, the hissing Pytho gives when it is killed by Apollo's arrows. By consequence, he has to set to music something that is properly not musical. 
Brillez et luisez encore une fois, ô jolis petits serpents d'or ! Une fois encore, faites entendre vos voix de clochettes!

\section{E. T. A. Hoffmann, Le Vase d'or'}

Si le serpent appartient bien à l'imaginaire occidental comme oriental, il n'a guère été porté sur la scène musicale par nos compositeurs. Les grands serpents de l'opéra n'ont pas de rôles qui mettent en valeur leurs attributs sifflants. Le terrible monstre de la Zauberflöte de Wolfgang Amadeus Mozart n'a d'existence que dans les cris d'angoisse de Tamino et ceux victorieux des Trois Dames ; point ne serait même besoin de lui sur scène. Quant au Fafnir du Siegfried de Richard Wagner, au lieu d'être une bête à la langue fourchue, c'est une voix de basse au langage plus qu'humain. Dans la musique moderne, on ne retiendra chez les reptiles que la démarche lancinante des tortues, suggérée par Camille Saint-Saëns dans son Carnaval des Animaux, par le malin détournement d'un air de Jacques Offenbach habituellement plus entraînant. À moins de voir dans les «Pianistes" de sournois musiciens, il faut renoncer.

C'est semble-t-il gageure de vouloir mettre en musique le cri du serpent, qui nous paraît se limiter à la consonne $[\mathrm{s}]$, alors même que ce sont les voyelles qui portent la mélodie. Les Grecs en étaient parfaitement conscients dans leur système de notation musicale. Et pourtant, ils ont tenu le pari de mettre en scène le sifflement du serpent, dans le cadre du nome pythique joué dans les concours delphiques. C'était même un passage obligé. La question est donc de savoir comment les Grecs ont pu rendre musical ce qui ne l'est pas fondamentalement. C'est à mon sens grâce à deux ambiguïtés dont le sifflement est porteur en Grèce que les horizons musicaux ont pu être ouverts. Dans cette mesure, j'explorerai d'abord le rapport entre le sifflement et la voix, puis entre le sifflement et le chant, afin de parvenir à comprendre dans un dernier temps comment l'on peut mettre en musique le sifflement.

1. Trad. Espiau de la Maëstre (Béguin 2002 : 18).

\section{LE SON INARTICULÉ : LE SIFFLEMENT ET LA VOIX}

\section{LA VOIX DES ANIMAUX}

Aristote consacre un petit chapitre de son Histoire des Animaux à la question de la voix, où il établit une distinction fondamentale qu'il est opportun de rappeler ici :

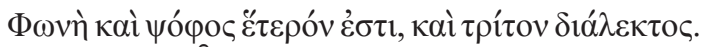

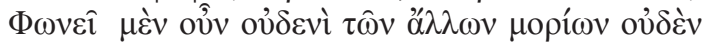

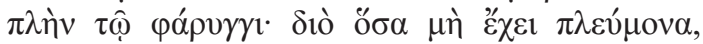

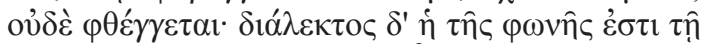

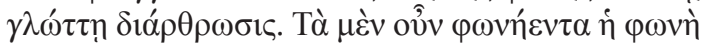

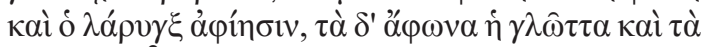

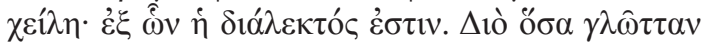

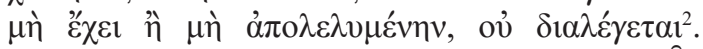

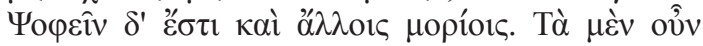

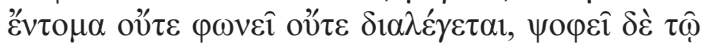

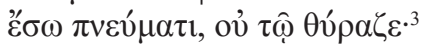

"La voix et le son sont deux choses différentes, le langage en est une troisième. On n'émet la voix par aucune autre partie que le pharynx ; c'est pourquoi tous les animaux qui n'ont pas de poumons n'ont pas de voix. Le langage est l'articulation de la voix au moyen de la langue. Donc les voyelles sont émises par la voix et le larynx, mais les consonnes le sont par la langue et les lèvres. Des unes et des autres procède le langage. C'est pourquoi tous les animaux qui n'ont pas de langue ou qui en ont une non déliée ne possèdent pas le langage. Mais on peut produire du son par d'autres parties du corps. Les insectes n'ont ni voix ni langage, mais ils produisent des sons avec l'air qu'ils ont à l'intérieur et non à l'extérieur. "

Les animaux peuvent donc se répartir en deux catégories selon le critère du bruit qu'ils produisent : certains ont de la voix - et l'organe nécessaire pour ce faire est le poumon -, les autres n'ont que le son pour eux. Il ne semble pas qu'un animal puisse rentrer dans les deux catégories à la fois : Aristote en fait donc un critère discriminant exclusif.

2. On retient ici l'édition donnée par la Bibliotheca Teubneriana, la Loeb Classical Library et la Collection des Universités de France.

3. Aristote, Histoire des animaux, IV, 9, 535a-b. Sauf mention contraire, toutes les traductions sont de l'auteur. 
Dans l'esprit d'Aristote, la dernière distinction, celle du langage, permet bien sûr d'isoler l'homme, dans la mesure où lui seul a cette maîtrise du langage, c'est-à-dire la capacité à articuler la voix en créant une différence entre ce qui relève de la voix, les voyelles, comme le montre l'étymologie grecque, et les consonnes qui sont en quelque sorte de la non-voix.

C'est le propre de la voix des animaux que d'être inarticulée. Le fait est confirmé par un texte d'Élias (VI ${ }^{\mathrm{e}}$ siècle ap. J.-C.) commentant l'Introduction de Porphyre (III siècle ap. J.-C.) :

ov̂ं

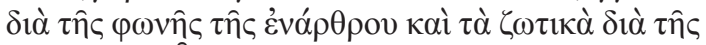

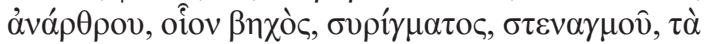

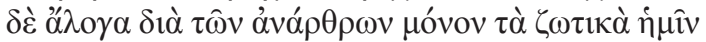

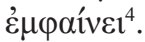

"Celui-ci, en effet, fait connaître ses affections cognitives par le moyen du son vocal articulé, ses affections biologiques par le moyen du son vocal inarticulé, comme la toux, le sifflement, le gémissement; les bêtes, en revanche, par le seul moyen du son vocal inarticulé, nous font voir leurs affections biologiques. »

C'est bien la clef de la distinction entre animal et homme que la question de l'articulation de la voix. On notera que dans les voix animales non articulées, Elias prend précisément l'exemple du sifflement, lorsque l'animal veut faire savoir qu'il souffre, mais le fait vaut bien sûr pour celui qui siffle naturellement, le serpent.

Articuler, c'est donc créer de la différence entre des voyelles et des consonnes, de la voix et de la non-voix : les consonnes pour exister n'ont pas besoin de souffle et pourraient ainsi être considérées comme de seuls sons, ce qu'Aristote toutefois ne dit pas explicitement. Notons également dès à présent que le siège des consonnes pour Aristote est la langue et les lèvres, ce qui se révèlera important pour le sifflement du serpent, auquel Aristote prête bel et bien une voix :

4. Élias, Commentaire sur l'Introduction de Porphyre, XXXVII, 5.

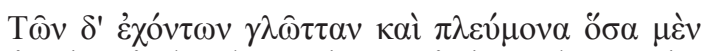

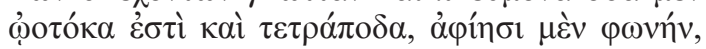

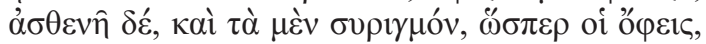

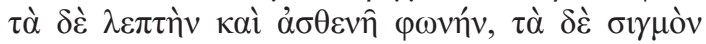

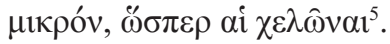

«Parmi les animaux qui ont une langue et des poumons, tous ceux qui sont ovipares et quadrupèdes émettent une voix, mais elle est faible ; les uns émettent un sifflement, comme les serpents, d'autres une voix douce et légère, d'autres un petit sifflement, comme les tortues."

Le sifflement est très clairement considéré par Aristote comme un type de voix ; par ailleurs, c'est bien un long sifflement, par opposition au petit sifflement des tortues, qui semble caractériser les serpents. La remarque d'Aristote se retrouve dans l'Histoire Naturelle de Pline l'Ancien, qui a traduit le maître grec tout en simplifiant le propos :

Oua parientibus sibilus, serpentibus longus, testudini abruptus.

"Les ovipares produisent un sifflement, long pour les serpents, mais bref pour la tortue."

\section{LE SIFFLEMENT}

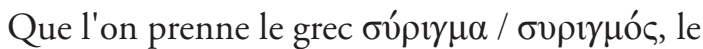
latin sibilus ou le français "sifflement ", on peut convenir que si l'on n'a pas de pures onomatopées, la consonne $[s]$ n'est pas étrangère à la formation de ces mots. Elle devait être associée au sifflement du serpent bien avant le fameux « Pour qui sont ces serpents qui sifflent sur vos têtes? » 7 .

Michel Psellos, auteur tardif mais dont les vues rejoignent souvent celles des plus anciens penseurs, explique ainsi dans un poème didactique que certaines consonnes de la langue grecque reposent sur un sifflement :

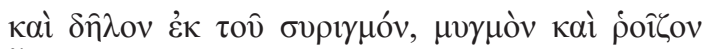
है $\chi \varepsilon ı$.

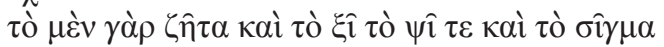

5. Aristote, Histoire des animaux, IV, 9, 536a.

6. Pline, Histoire naturelle, XI, 267.

7. J. Racine, Andromaque, acte V, scène 5, v. 1638. 


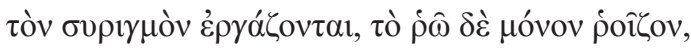

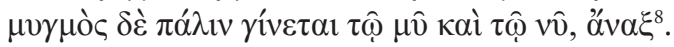

" D'où il est évident qu'il y a le sifflement, le murmure et le ronflement :

le zèta, le xi, le psi et le sigma

produisent le sifflement, le rhô seul produit le ronflement,

en revanche, ô prince, le murmure appartient au mu et au nu. "

Il reprend ici des éléments déjà connus de Denys d'Halicarnasse, qui, dans son traité sur l'agencement des mots, consacre de longs développements aux sonorités. Il s'interroge ainsi sur ce qui produit les différents sons, préalable nécessaire avant l'étude du rythme et de la mélodie de la phrase. Denys prend en compte les éléments anatomiques nécessaires à la production du sifflement - langue, palais, dents - ; il ne s'agit pas là d'une remarque anodine, car c'est ce que les Grecs ont tendance à retenir du serpent anatomiquement : ses dents acérées et sa langue bifide sont particulièrement marquantes. Il faut donc garder à l'esprit que ces éléments sont associés à la production du sifflement :

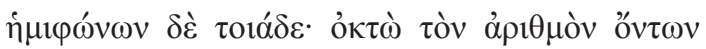

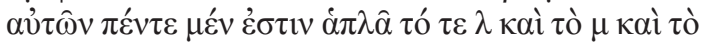

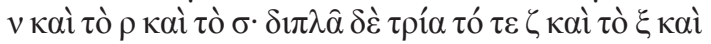

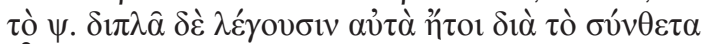

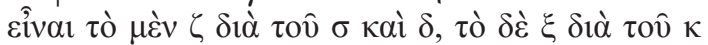

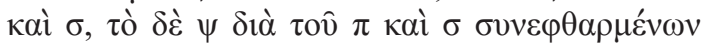

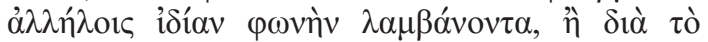

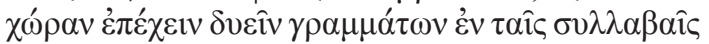

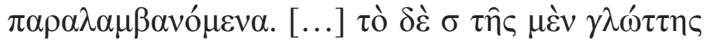

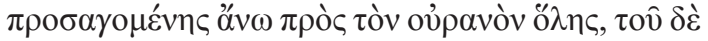

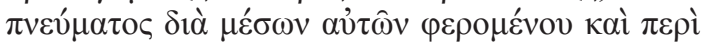

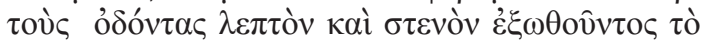
бó $\rho \gamma \mu \alpha^{9}$.

"Voici les semi-voyelles : elles sont au nombre de huit et cinq d'entre elles sont des semi-voyelles simples : le lambda, le mu, le nu, le rhô et le sigma. Il y en a aussi trois qui sont des semi-voyelles doubles, le zêta, le xi et le psi ; on les dit doubles parce que le zêta est composé d'un sigma et d'un delta, le xi du kappa et du sigma, le psi du pi et du

8. Michel Psellos, Poèmes, VI, 260-263.

9. Denys d'Halicarnasse, Composition stylistique, VI, 14, 14 et 17. sigma, et que, comme les deux lettres sont confondues ensemble, elles ont un son particulier, ou parce que dans les syllabes elles occupent la place de deux lettres. [...] Le sigma s'obtient en montant la langue tout entière vers le palais, en poussant le souffle entre les deux et en émettant un sifflement léger et ténu au niveau des dents. "

Autre point que développe Denys, c'est le groupe de lettres auquel le sigma appartient : consonne ou voyelle ? Denys introduit une catégorie surprenante et par là même une précision fondamentale : le sigma et toutes les lettres qui en sont parentes, comme le relève Psellos, appartiennent à un groupe qui n'est ni tout à fait les voyelles, ni tout à fait les consonnes. Ce sont les hémiphôna, les semi-voyelles. L'expression a de quoi surprendre, surtout lorsque l'on pense au sifflement sourd qu'évoque pour nous le sigma. Comment s'imaginer que l'on est à mi-chemin entre une voyelle et une consonne ? On comprend alors qu'il ne puisse pas y avoir de contradiction à dire du serpent qu'il a une voix, alors même que ce terme semble plutôt se référer aux voyelles qu'aux consonnes. Mais là où nous devons reprendre notre jugement, c'est que ce qui nous semble consonne peut appartenir pour les Grecs à une catégorie qui laisse en quelque sorte la place à une certaine vocalisation de la consonne.

Denys ne s'en justifie pas et reprend en fait une classification déjà existante, que l'on trouve déjà dans la Poétique d'Aristote ${ }^{10}$ et que l'on retrouve dans le traité sur la musique d'Aristide Quintilien :

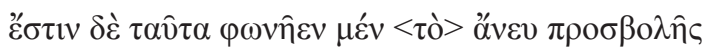

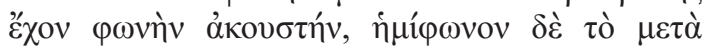

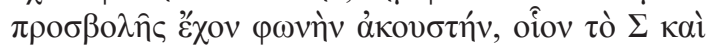

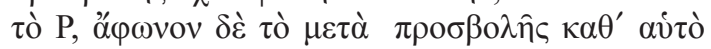

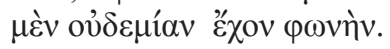

"La voyelle est ce qui a un son audible sans attaque, la semi-voyelle ce qui a un son audible avec attaque, par exemple le sigma et le rhô, et la consonne ce qui n'a aucun son en soi avec attaque. "

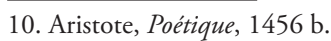




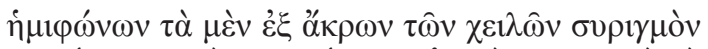

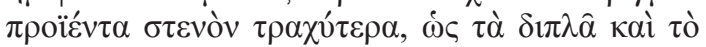

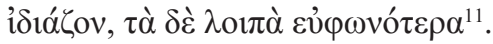

"Parmi les semi-voyelles, les unes produisent assez rudement un bref sifflement de la pointe des lèvres, qu'elles soient doubles ou simples, et les autres ont un plus beau son. "

On y apprend que certes le sigma est une semivoyelle, mais rentre dans une sous-catégorie des semi-voyelles rauques, peu mélodieuses. On constate également qu'Aristide Quintilien attache plus d'importance aux lèvres, comme si le sifflement venait uniquement d'elles.

\section{SifFLEMENT ET RAISON}

Quoique le sifflement puisse porter une part de voyelle, il n'est pas pour autant une articulation entre voyelle et consonne et donc il n'est pas en soi l'expression d'un langage. C'est clairement ce qu'exprime Grégoire de Nysse dans le Contre Eunome:

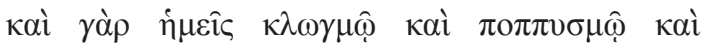

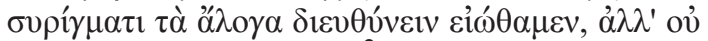

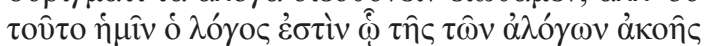

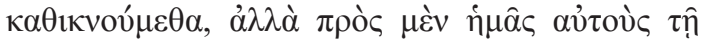

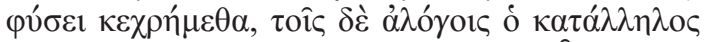

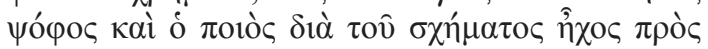

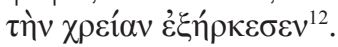

"En effet, nous avons l'habitude de diriger les êtres sans raison par un gloussement, un appel de la langue ou un sifflement : ce n'est pas par le langage rationnel que nous parvenons à nous faire entendre des êtres sans raison; envers nous-mêmes nous usons de la nature, mais aux êtres sans raison suffisent le son qui leur correspond et tel ou tel bruit, si besoin est. "

Le sifflement, comme les claquements de langue et autres effets sonores sont réservés aux bêtes, car elles ne sauraient comprendre davantage. L'homme en quelque sorte est dans un stade régressif quand il s'adresse aux bêtes. Il suffit pour s'en faire obéir d'imiter le son qui leur est propre :

11. Aristide Quintilien, Traité sur la musique, II, 11.

12. Grégoire de Nysse, Contre Eunome, II, 1, 421.

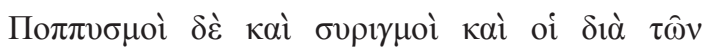

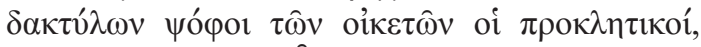

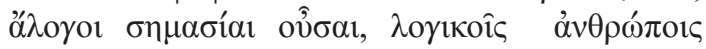
$\dot{\varepsilon} \kappa \kappa \lambda \imath \tau \dot{\varepsilon} \mathrm{ol}^{13}$.

"Les appels de la langue, les sifflements et les bruits de doigts qui servent à appeler leurs domestiques, puisque ce sont des signes irrationnels, les hommes doués de raison doivent les éviter. »

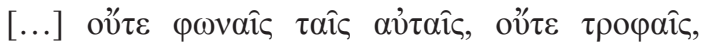

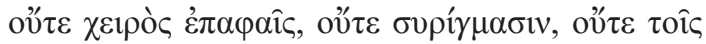

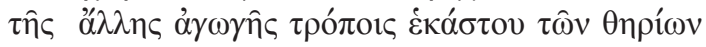

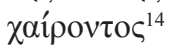

" [...] puisque chaque bête sauvage n'a pas plaisir aux mêmes voix, aux mêmes aliments, aux mêmes contacts de la main, aux mêmes sifflements ni aux mêmes gestes d'autre sorte ..."

Le témoignage de Clément d'Alexandrie ou encore de Grégoire de Nazianze va dans le même sens que celui de Grégoire de Nysse : les sons auxquels les hommes recourent pour faire marcher les bêtes ne sont que des signes dénués de raison, sinon celle que l'homme a en tête quand il veut produire un effet sur un animal. En tous les cas, selon l'Alexandrin, il ne sied pas à des hommes de recourir à ce type de sons. Dans ces deux témoignages, il s'agit surtout des animaux domestiques et le sifflement dont il est question ne saurait s'adresser au serpent, mais ils montrent la perception que les Grecs ont de ce type de sonorité dans leur système de valeurs. Le sifflement est donc dénué de toute forme de raison ou de sens. Comme les définitions d'Eustathe ou de la Souda le précisent, il appartient proprement au monde de la bête, sauvage même, voire monstrueuse :

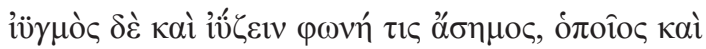

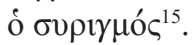

"Le gémissement, gémir, ce sont des voix sans signification, exactement comme le sifflement. "

13. Clément d'Alexandrie, Le pédagogue, II, 7, 60.

14. Grégoire de Nazianze, Discours apologétique (= Disc. 2), $44=452 \mathrm{c}$.

15. Eustathe, Commentaire à l'Iliade, IV, 261. 
Perrot S.

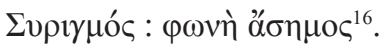

"Sifflement : voix sans signification. »

Toutefois, si en soi le sifflement n'a pas de sens, l'homme lui prête une signification. En effet, lorsqu'il entend un sifflement, l'homme associe presque inévitablement ce son au danger. Pour Grégoire de Nazianze, le sifflement semble aller de pair avec le venin de l'animal : le sifflement est alors perçu comme un signal d'une attaque imminente :

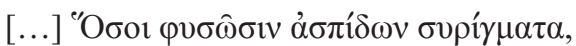

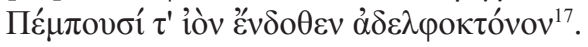

«[... tous ceux qui parmi les cobras exhalent des sifflements

Et envoient de l'intérieur un venin fratricide. »

Michel Psellos de son côté associe le sifflement au danger dans le cadre de la chute de la foudre : le sifflement n'est pas dénué ici d'une certaine puissance sonore.

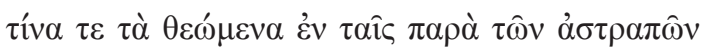

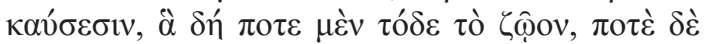

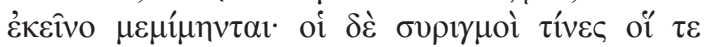

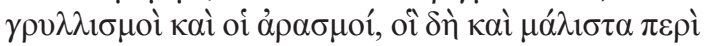

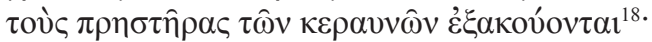

«Et quels spectacles dans les déflagrations des éclairs, qui imitent tantôt cet animal, tantôt cet autre : des sortes de sifflements, grognements et coups de sabots contre terre, que l'on entend précisément lors des ouragans où tombe la foudre. "

Mais c'est bien sûr avant tout le sifflement du serpent qui fait naître la crainte d'un danger imminent. On peut se reporter aussi bien à une scholie de l'Iliade qu'à un texte de Diodore de Sicile qui décrit le sifflement d'un serpent comme surnaturel :

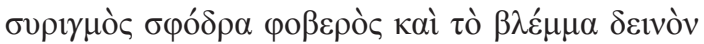

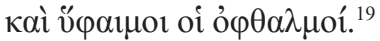

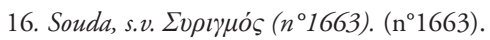

17. Grégoire de Nazianze, Poèmes, 1185, 3-4.

18. Michel Psellos, Sur la météorologie = Opuscules 19, 17.

19. Scholies à l'Iliade, XXII, 95.
« un sifflement fort effroyable, un regard terrible et des yeux injectés de sang »

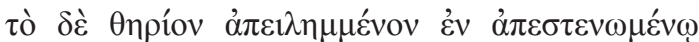

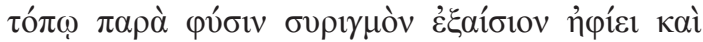

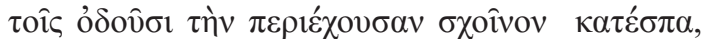

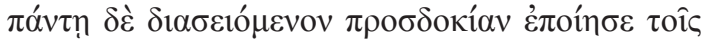

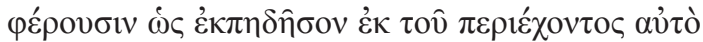

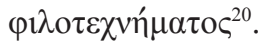

"La bête, bloquée dans un espace restreint, poussait des sifflements contre nature, extraordinaires, et de ses dents essayait de disloquer le panier de jonc qui la contenait, et, s'agitant en tous sens, elle faisait craindre à ceux qui la portaient qu'elle échapperait au piège qui la contenait. "

Élien parle quant à lui des sifflements d'un serpent qui suffisent à effrayer tous les habitants de l'île de Chios :

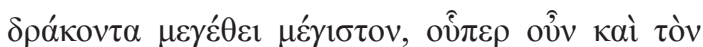

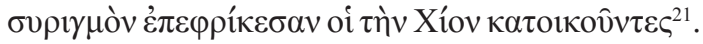

" un serpent très grand par la taille, dont le sifflement faisait frémir les habitants de Chios. »

Ajoutons enfin le témoignage de Philostrate qui, dans la Vie d'Apollonios de Tyane, évoque les horribles sifflements des serpents qui infestent les rivages de la mer Rouge ${ }^{22}$. C'est le même type de sifflement qu'Élien décrit dans son traité sur La personnalité des animaux ${ }^{23}$.

Depuis Aristote jusqu'à la Souda, le sifflement est donc considéré comme une voix mais l'on peut se demander si le sifflement est la seule voix du serpent. Le texte d'Aristote précise que les ovipares ont une voix faible et pour certains un plus ou moins long sifflement. Il reste donc à savoir si le potentiel musical du serpent ne repose pas sur cette ambiguïté. En effet, le serpent ne semble pas complètement étranger au monde musical.

20. Diodore de Sicile, Bibliothèque historique, III, 37, 6.

21. Élien, Personnalité des animaux, XVI, 39.

22. Philostrate, Vie d'Apollonios de Tyane, III, 8, 26.

23. Élien, Personnalité des animaux, XV, 21. 


\section{LE SERPENT, UN ANIMAL MUSICIEN ?}

\section{LE SERPENT AIME-T-IL LA MUSIQUE ?}

L'imaginaire occidental s'est souvent complu à représenter le personnage du charmeur de serpent : les voyageurs européens restaient subjugués par la manière dont les serpents semblaient obéir à la mélodie lancinante de leur maître flûtiste. Le personnage du charmeur de serpent est devenu une image d'Épinal de l'art occidental. Il suffit de penser à la Charmeuse de serpents d'Henri Rousseau ou au Charmeur de serpents de Jean-Léon Gérôme.

Mais le charmeur de serpents, on l'a bien compris depuis, est un grand illusionniste. Alors même que le public est persuadé que le reptile réagit à la mélodie, il est en fait bien plus attentif au mouvement de l'instrument, qui d'ailleurs n'est pas nécessairement lié à la mélodie jouée. De même, le public ne fait guère attention au pied du musicien qui discrètement donne des impulsions : c'est à ces vibrations du sol ainsi produites que le serpent est sensible et c'est ce petit tour qui le rend attentif ensuite au balancement de la flûte. Le serpent en effet est un animal sourd au sens où nous l'entendons : la morphologie de son oreille ne lui permet de saisir que très peu de sons ${ }^{24}$.

Nous connaissons aujourd'hui des serpents qui disposent d'un autre type de moyen pour produire du son : les serpents à sonnette, qui tirent leur nom de leur bruiteur, la cascabelle, qui est un assemblage de grandes écailles en anneaux imparfaitement fixées. Presque toutes les espèces du genre Crotalus en ont un. Ce genre emprunte son nom à un instrument de percussion grec, les crotales, qui consistent en deux morceaux de bois ou de bronze qu'on tape l'un contre l'autre. Mais les Grecs ne sauraient en être responsables, puisque ces ophidiens ne se trouvent qu'en Amérique du Nord et centrale.

24. Voir Platel (2005 : 51-52), qui conclut : « L'audition n'est donc pas absente de l'univers perceptif des serpents, mais elle n'y joue pas un rôle essentiel ».
Dans l'Antiquité, on considère donc que le serpent est plus réceptif aux autres sifflements qu’à la musique à proprement parler. Le sifflement fonctionne même comme un code entre les serpents, voire avec les autres espèces de reptile. Le serpent se sert tout d'abord de son sifflement pour exprimer son autorité sur le groupe : on peut se référer à une scholie d'Eschyle, où il est écrit qu'un serpent fait taire, par son cri poussé à midi, tous les autres serpents à la ronde. Il est intéressant de voir ici qu'Eschyle emploie le terme de ßoóc pour désigner le cri du serpent, ce qui est assez unique et que commente la scholie :

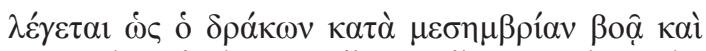

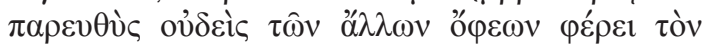

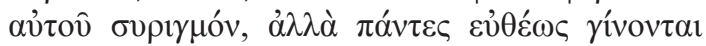
$\dot{\alpha} \varphi \alpha v \varepsilon \hat{\varepsilon} \varsigma^{25}$.

« Il est dit que le serpent crie à midi et aussitôt aucun des autres serpents ne supporte son sifflement, et tous sitôt de disparaître. "

Le sifflement du serpent est aussi porteur d'un code nuptial : il est un appel à l'accouplement. Ainsi Épiphane dans son ouvrage sur les Héré-

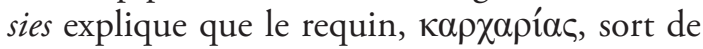
la mer pour appeler la vipère ${ }^{26}$ :

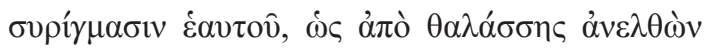

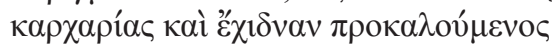

«par ses sifflements, comme lorsque le requin sort de la mer et appelle la vipère"

Une scholie à Oppien mentionne pour sa part le fait que la murène sort rapidement de la mer quand elle entend le sifflement du serpent pour s'unir à lui :

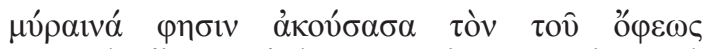

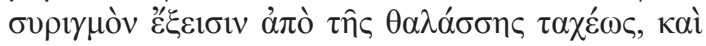

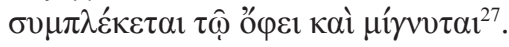

" On dit que la murène, entendant le sifflement du serpent, sort rapidement de la mer, s'enroule autour de lui et s'unit à lui. "

\footnotetext{
25. Scholies aux Sept contre Thèbes d'Eschyle, 381.

26. Épiphane, Panarion. Contre les hérésies, I, 463, 26.

27. Scholies aux Halieutiques d'Oppien, 564.
} 
On peut douter de la réalité de ces remarques dans le règne animal, mais ce qui est sûr, c'est que pour les Anciens, le sifflement du serpent peut fonctionner comme un élément de parade nuptiale.

Peut-on pour autant parler de chant ? On serait tenté de répondre par la négative, mais les Anciens n'hésitaient pas à parler de chant des cigales, alors qu'Aristote reconnaît lui-même qu'il n'y a de chant que par métaphore; encore le fait-il à mots couverts :

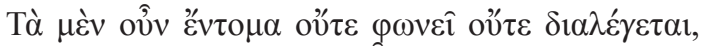

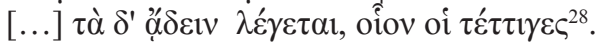

"Les insectes n'ont ni voix ni langage, $[\ldots]$ mais on dit de certains qu'ils chantent, ainsi les cigales. »

Or le chant des cigales, nous pourrions le transcrire par la lettre xi, qui précisément, comme on l'a vu, est faite d'un sifflement. Mais force est de constater que si l'on associe volontiers la cigale au monde de la musique en Grèce, au point d'en avoir fait un récit étiologique, nous n'avons aucune source pour nous parler d'un chant du serpent.

\section{Potentialités D'Un CHANT DU SERPENT}

La raison en est peut-être que dans l'Antiquité, certains penseurs estiment que le sifflement n'est pas une sonorité esthétiquement belle. Le témoignage de Denys d'Halicarnasse est tout à fait révélateur de ce point de vue :

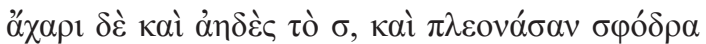

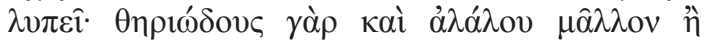

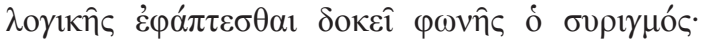

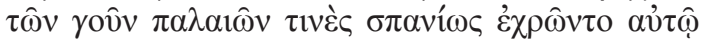

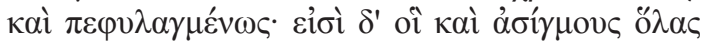

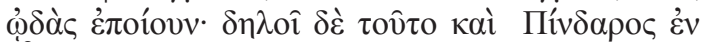

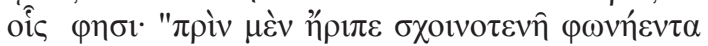

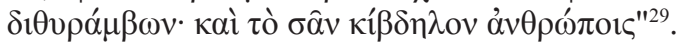

"Le sigma n’a ni grâce ni agrément, et utilisé avec excès, il chagrine fortement l'oreille : le sifflement donne l'impression de relever du sauvage et de l'irrationnel plutôt que de la voix pleine de raison. Chez

28. Aristote, Histoire des animaux, IV, 9, 535b.

29. Denys d'Halicarnasse, Composition stylistique, VI, 14, 20. les Anciens, on en usait rarement et avec retenue ; il y en a même qui ont composé des odes entières sans sigma. Le fait est attesté par Pindare quand il écrit : "autrefois, se trainnaient, déroulées, les voyelles des dithyrambes; et le san était de mauvais aloi pour les hommes" ".

Et pourtant, le vocabulaire musical peut être mis à contribution quand est considérée la voix du serpent. Ainsi dans ses Cynégétiques, Oppien évoque certes la bête venimeuse et ses sifflements, mais emploie un autre verbe pour désigner les sons que le serpent produit avec son corps du serpent : il nous dit en effet qu'il choque son menton, qu'il fait un bruit de percussion avec lui tout en sifflant :

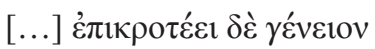

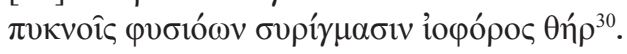

"[...] la bête venimeuse choque son menton en respirant bruyamment de ses sifflements resserrés. "

Il n'est pas aisé de savoir de quoi il s'agit exactement, mais le verbe grec est sans ambiguïté : il s'agit bien d'un bruit de percussion - c'est la racine de « crotale ».

Nonnos de Panopolis de son côté compare le cou du serpent à une salpinx, instrument antique équivalent de notre trompette, qui émet des sons, très précisément un sifflement :

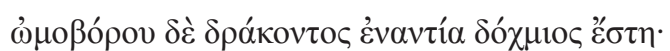

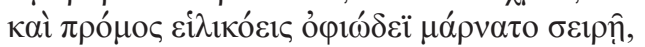

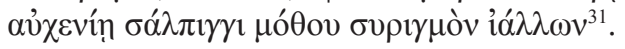

« [Damasen] fit face au serpent carnassier en l'abordant par le côté

et le champion aux replis sinueux lutta avec sa chaîne serpentine,

poussant un sifflement belliqueux avec la trompette de son cou. "

Même si c'est là un jeu métaphorique de la poésie épique, il est intéressant de noter que l'on recourt à un instrument de musique pour définir le cri du serpent.

30. Oppien, Cynégétiques, II, 244-245.

31. Nonnos de Panopolis, Dionysiaques, XXV, 501-503. 


\section{L'AMBIGUÏTÉ DU SIFFLEMENT}

Un oracle sibyllin met en lien, par le jeu de la poésie, le sifflement qui apparaît comme la typique imitation du bruit du serpent et une fois encore la salpinx :

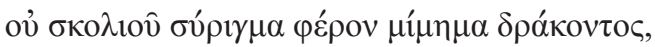

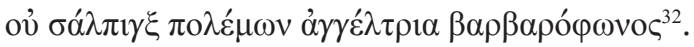

"Pas de sifflement faisant l'imitation du serpent tortueux, Ni la salpinx au son barbare annonciatrice des guerres. "

Par ailleurs, l'auditeur de ces vers ne peut pas ne pas penser au mot syrinx, en entendant d'un côté les syrigmata et de l'autre la salpinx. Or il n'est bien sûr pas du tout question de flûte de Pan dans ces vers, qui pourtant créent un univers sonore qui y fait penser. Par ailleurs, la salpinx n'est pas présentée sous son meilleur jour : c'est l'instrument à la voix barbare, qui annonce les guerres; les sifflements du serpent sont tout aussi funestes. Inversement, la syrinx est l'instrument de la paix, c'est la musique des bergers qui surveillent leurs troupeaux. C'est que la racine sur- en grec est porteuse naturellement d'une certaine ambiguïté. Comme l'écrit Fr. Skoda, " ces formes grecques en sur-peuvent donc traduire autant le son produit par le resserrement des lèvres pour siffler quelqu'un ou siffloter une mélodie, que le sifflement rendu par des sifflantes dentales sourdes. " (Skoda 1983 : 383) Le sifflement grec porte en lui cette tension entre la non-musicalité et au contraire l'ouverture vers la musique.

Ainsi le sifflement peut-il être associé curieusement à ce qu'il y a de plus mélodieux, le chant du cygne (Arnott 1977), même si c'est là une attestation très tardive, puisqu'elle n'apparaît que sous le calame de Grégoire de Nazianze. Dans un poème autobiographique où le théologien voit sa mort approcher, il dit ne trouver que la consolation de la comparaison avec le cygne qui entonne son plus beau chant à sa mort. Platon dans le Phédon ${ }^{33}$ expliquait que

32. Oracles Sibyllins, VIII, 116.

33. Platon, Phédon, 84e-85b. ce n'est pas là un chant funèbre, exprimant la tristesse de quitter la vie, mais la joie de gagner le monde divin. Le théologien chrétien s'inscrit dans cette même lignée : le dernier chant du cygne n'est pas un thrène, un chant de deuil, mais un chant de départ ${ }^{34}$ :

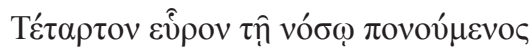

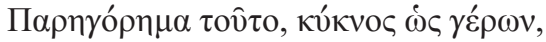

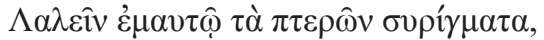

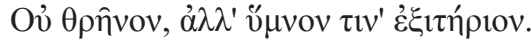

"Quatrièmement, j'ai trouvé, alors que je souffre de la maladie, cette consolation, comme le vieux cygne, prononcer pour moi-même les sifflements des ailes, non le thrène, mais l'hymne de départ. "

On comprend par ailleurs que la perspective chrétienne de l'au-delà favorise cette interprétation du chant du cygne. Mais ce qui est intéressant ici, ce n'est pas tant le chant de l'animal que le bruit que font ses ailes déployées, très clairement un sifflement :

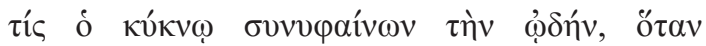

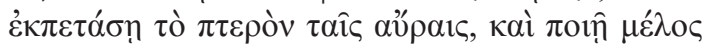
$\tau o ̀ ~ \sigma u ́ \rho t \gamma \mu \alpha ;^{35}$

"Qui est celui qui tisse son chant avec le cygne, quand il déploie son aile dans les brises et fait du sifflement une mélodie?»

Ce dernier peut donc accompagner le plus beau de tous les chants sans que la musique s'en trouve lésée, puisque, si le chant est mélodieux, le bruit des ailes l'est tout autant, au point que l'on peut se demander si ce n'est pas ce sifflement qui est en fait le fameux chant du cygne.

On le voit donc bien, le sifflement est porteur d'une grande ambiguïté dans sa réalisation : sourd, il n'est crédité d'aucun intérêt esthétique ; sonore, il ouvre des perspectives musicales. Mais pour bien saisir les enjeux musicaux de cette question, il convient de revenir sur ce qui définit l'art musical pour les Grecs.

34. Grégoire de Nazianze, Poèmes, 1333, 6.

35. Grégoire de Nazianze, Discours théologique (= Disc. 28), XXIV (= 60a). 


\section{SIFFLEMENT ET IMITATION : LA CRÉATION MUSICALE}

\section{L'IMITATION EN MUSIQUE}

La pratique musicale grecque était intimement liée à la notion d'imitation, comme la plupart des autres arts. Il s'agit d'une imitation au sens large vraisemblablement : on peut imiter par la musique une scène, c'est-à-dire suggérer par la musique quelque chose qui relève normalement du visuel, mais on peut aussi tâcher de faire naître par la musique des émotions qu'on aurait devant tel ou tel spectacle. C'est une problématique qui a été particulièrement développée au $\mathrm{XIX}^{\mathrm{e}}$ siècle, dans le cadre de la réflexion sur le poème symphonique. On pensera bien sûr aux fameux Tableaux d'une exposition de Modeste Moussorgsky. Quant à mettre des animaux sur la scène musicale, Franz Anton Schuber ${ }^{36}$ et Nikolaï Rimski-Korsakov s'y sont essayés respectivement avec l'abeille et le bourdon, et ils seront suivis par le Pierre et le Loup de Sergueï Prokofiev ou encore l'Oiseau de Feu d'Igor Stravinsky.

Un texte de Denys d'Halicarnasse montre combien l'imitation joue un rôle majeur dans la culture grecque :

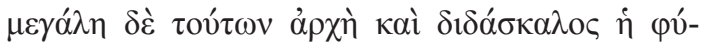

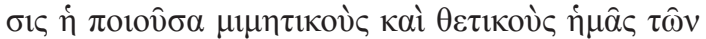

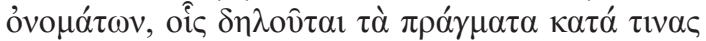

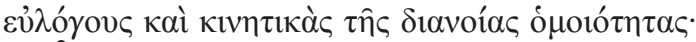

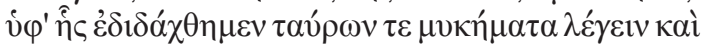

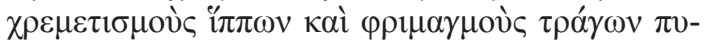

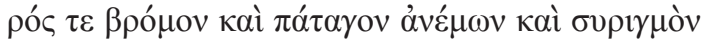

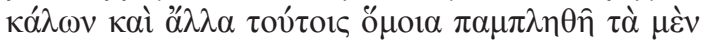

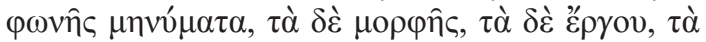

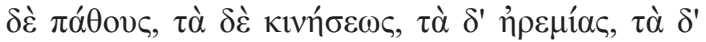

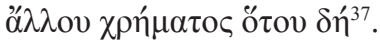

"Le grand principe et le maître de tout cela, c'est la nature, qui nous fait imitateurs et créateurs de

36. On se gardera de confondre ce compositeur originaire de Dresde avec son illustre homonyme autrichien.

37. Denys d'Halicarnasse, Composition stylistique, VI, 16, 2-3. noms, qui décrivent les choses selon des associations d'idées rationnelles ou émotionnelles. C'est elle qui nous apprend à dire les mugissements des taureaux, les hennissements des chevaux, les grognements des boucs, le grondement du feu, le fracas des vents, le sifflement des cordages, et à utiliser tant d'autres termes du même genre qui imitent une voix, une silhouette, une action, une émotion, le mouvement, la détente, ou n'importe quoi d'autre. "

Afin de rendre compte de la nature, l'homme doit mettre un nom sur chaque chose et selon Denys, un des procédés les plus évidents, c'est imiter le bruit que l'on entend, en procédant par onomatopée. Après quelques exemples d'animaux, il parle du sifflement qui est par exemple celui des cordages d'un navire. Sifflement de serpent ou de cordages, ce bruit trouve une manière d'être dit par imitation. Si Denys décrit là un processus linguistique, la musique procède de la même manière. Nous disposons à ce titre de deux exemples significatifs qui font intervenir le serpent.

\section{LES DERNIERS SIFFLEMENTS D'UN MONSTRE}

Les textes anciens nous ont laissé le nom de deux compositions musicales, qui présentaient la particularité d'avoir un programme imposé, ce qui leur valait d'être rangées dans la catégorie des nomes. C'étaient en quelque sorte des "sonates à programme " (Bélis 1999 : 131). Le contenu narratif en était déjà décidé auparavant mais la mise au point des mélodies et le détail du texte (s'il y avait lieu) étaient à la libre appréciation du musicien-compositeur.

Les deux nomes qui nous intéressent ici sont le nome pythique et le nome polycéphale. Le premier était l'épreuve-phare des concours des Pythia de Delphes, interprété à l'aulos seul mais aussi à la cithare et au chant, et enfin à la cithare seule. Le nome polycéphale nous est surtout connu par la XII e Pythique, où Pindare évoque l'invention de ce nome par la déesse Athéna, en même temps que celle de l'aulos. Les deux nomes ont en commun de raconter le combat d'un être divin ou chéri des dieux contre un 
monstre qui tient du serpent - Apollon contre Python et Persée contre Méduse -, le tout en musique.

C'est sans aucun doute sur le nome pythique que nous sommes le mieux renseignés. Nous disposons en effet de quatre sources, d'époques et de contextes différents, qui proposent une description de ce morceau. Il s'agit tout d'abord d'une scholie de Pindare, qui aborde le nome pythique dans la présentation de l'épreuve à laquelle aurait participé Midas d'Agrigente, l'aulète célébré par la XII Pythique. C'est ensuite un passage de Strabon, dans un des livres de sa Géographie qu'il consacre à la Grèce, dont le texte est assez mal transmis. Les papyrus d'Herculanum nous ont conservé le témoignage fragmentaire du philosophe épicurien Démétrios Lacon sur le nome pythique. Enfin, le lexicographe Pollux consacre une entrée de son Onomasticon à cette question, à la suite d'un développement sur l'aulos. Cependant, il faut d'emblée noter que ces sources ne sont pas d'accord entre elles pour la division du nome, mais ce n'est pas le lieu de faire cette conciliation.

La mort de Python est au cœur de la mythologie delphique, mais elle est aussi au cour de la musique delphique. C'est du moins ce que semblent indiquer les sources qui évoquent des prestations musicales dans le cadre des concours pythiques. En effet, dans les Hymnes à notation musicale gravés sur le Trésor des Athéniens, les artistes athéniens célèbrent Apollon en rappelant, en vers, de quelle manière ils l'ont déjà glorifié dans le cadre du concours. Même si on ne trouve que quelques vers à ce sujet, il est intéressant de remarquer qu'ils sont communs aux deux hymnes conservés. On y reconnaît aisément la description des deux grands concours delphiques, les Pythia et les Sôteria. L'allusion aux Pythia est évidente, et elle est présentée dans les termes suivants, selon l'édition et la proposition de traduction d'A. Bélis (Bélis 1992), respectivement dans le premier et le second hymne :

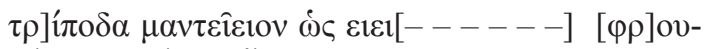

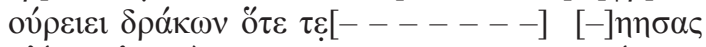

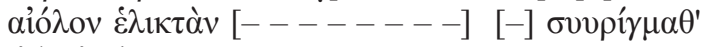
iicic $\dot{\alpha} \theta \omega ́ \pi \varepsilon\left[v \tau^{\prime}-----\right]^{38}$

" (Ils disent) comment tu conquis le trépied prophétique $[. .$.$] que gardait$

le serpent, lorsque de (tes traits, tu transperças) le monstre sinueux aux changeants replis,

et (comment) il (expira) en poussant d'effroyables sifflements $[. . .$.$] "$

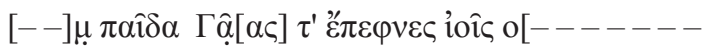

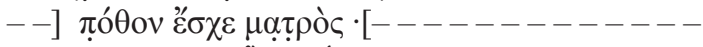

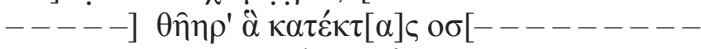

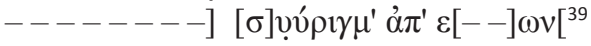

" Mais, (fils de) Létô, [dieu] (à l'aimable regard), ... et tu transperças de tes flèches la (farouche) enfant de la Terre $[\ldots]$; et elle fut saisie du désir de sa mère [...] ; tu massacras la bête (...), poussant un sifflement $[\ldots]$ »

Le genre musical retenu par les technites dans la glorification de leurs exploits impose une certaine concision, notamment dans le cas du second hymne qui se présente sous forme de strophes. Sont donc indiqués les éléments narratifs fondamentaux pour comprendre de quoi il s'agit. Même si le texte est mutilé, il ne saurait être démesurément plus long. En l'occurrence, les technites d'Athènes mettent l'accent sur le caractère monstrueux du serpent et sur son lien avec la Terre ; le combat lui-même est bref, limité aux seuls coups du dieu ; fait intéressant, les deux hymnes insistent sur la manière dont meurt le monstre, ses terribles sifflements. C'est sans doute là ce qui faisait le cour narratif et partiellement descriptif du nome pythique.

Nous avons dans ces deux hymnes très précisément la mise en musique de ces sifflements, mais l'épisode est extrêmement bref. Toutefois, fait inestimable, nous avons conservé la musique de ces passages (Fig. 1-2) : elle ne donne pas l'impression de vouloir faire ressortir

38. Athenaios, fils d'Athenaios, Péan à Apollon, strophe III, v. 21-24. 39. Limènios, fils de Thoinos, Péan et prosodion à Apollon, strophe VIII, v. 27-30. 
le sifflement en particulier, mais plutôt le sentiment d'angoisse qui naît de la bataille entre Apollon et Python. Musicalement, nous avons dans l'hymne de Limènios des sauts de septième qui traduisent l'instabilité du combat. Dans les deux hymnes, le mot syrigma est mis en valeur par le dédoublement des voyelles que fait entendre le changement de notes de musique : la mélodie est clairement sinueuse, tortueuse.

Mais ce n'est là qu'un lointain écho du nome pythique dont on sait, et nos quatre sources s'accordent sur ce point, qu'une des cinq parties canoniques était consacrée à la mort du serpent et qu'elle s'appelait syrigma selon le scholiaste de Pindare, syrigges selon Strabon, hyposyrigmos selon Démétrios Lacon (Rispoli 2007 : 86-87) et odontismos (en ce cas plutôt grincement de dent) selon Pollux :

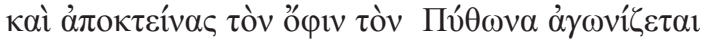

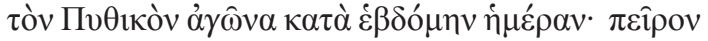

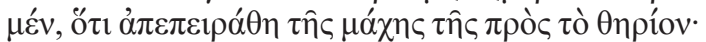

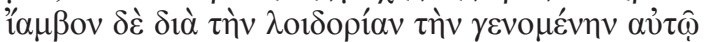

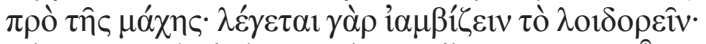

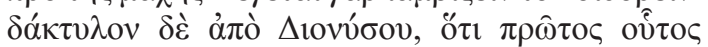

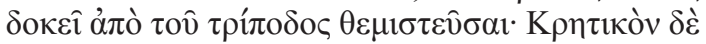

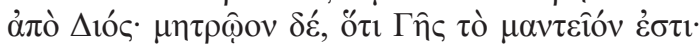

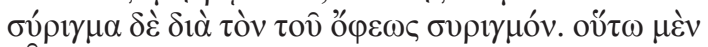

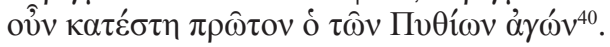

"Après avoir tué le serpent Python, il dispute le concours pythique le septième jour; il y a le peiron, parce qu'il a engagé le combat contre la bête ; l'iambon à cause des injures qu'il lui adresse avant le combat : on parle en effet d'iambizein (faire des iambes) pour loidorein (injurier); le daktylon vient de Dionysos, car il semble que ce soit le premier à avoir pratiqué la divination sur le trépied ; le krètikon vient de Zeus; le mètrôon, parce que l'oracle appartenait à la Terre; le syrigma à cause du sifflement du serpent. Ainsi fut instauré pour la première fois le concours pythique. »

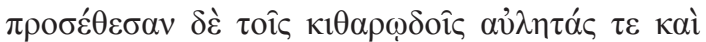

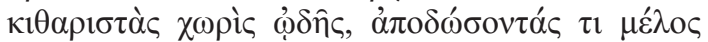

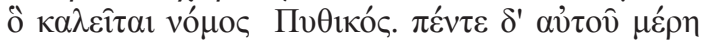

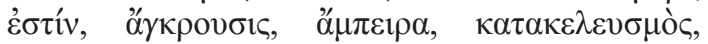

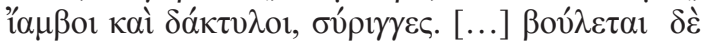

40. Scholies aux Pythiques de Pindare, XII, hyp. 24-32.

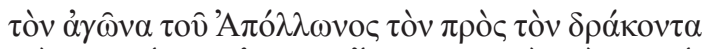

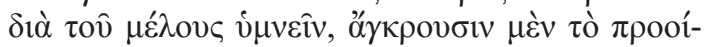

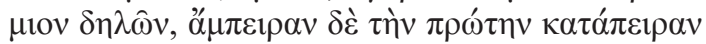

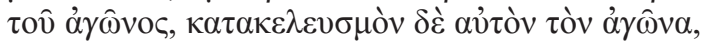

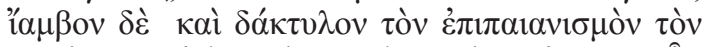

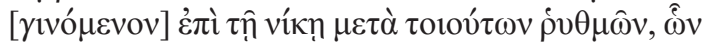

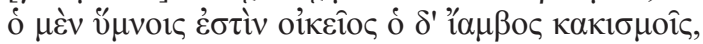

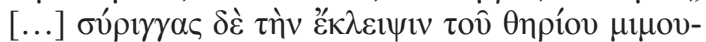

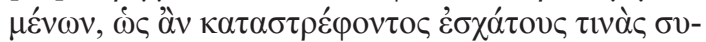
prypoúc $\varsigma^{41}$.

« Ils ajoutèrent aux citharodes des aulètes et des citharistes sans chant pour interpréter une composition musicale qu'on appelle le nome pythique. Il y a cinq parties : l'ankrousis (prélude), l'ampeira (attaque), le katakeleusmos (invective), les iambes et dactyles et les syringes (sifflements). [...] Il prétend célébrer par la mélodie le combat d'Apollon contre le serpent ; il fait entendre dans l'ankrousis le prélude, dans l'ampeira le premier assaut du combat, dans le katakeleusmos le combat luimême, dans l'iambe et dactyle le péan qui célèbre la victoire en usant de tels rythmes - le dactyle est propre aux hymnes et l'iambe aux reproches,$-[\ldots]$ dans les syringes l'imitation de l'expiration de la bête, comme si elle poussait ses tout derniers sifflements. »

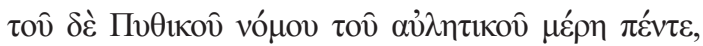

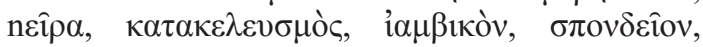

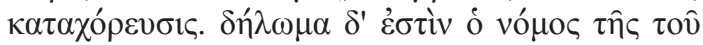

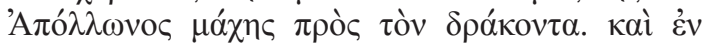

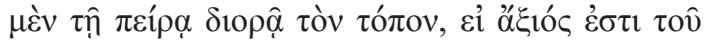

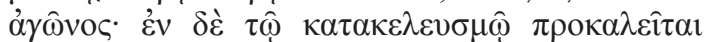

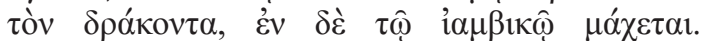

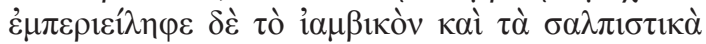

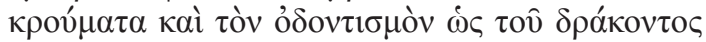

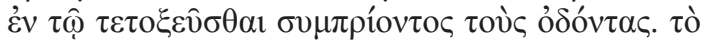

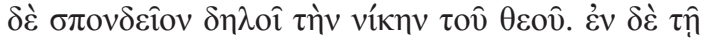

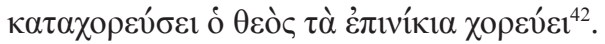

"Le nome pythique joué à l'aulos comporte cinq parties : la peira (exploration), le katakeleusmos (invective), l'iambikon (iambique), le spondeion (chant de libation) et la katachoreusis (danse de victoire). Le nome est la représentation du combat d'Apollon contre le serpent. Dans la peira, il explore le territoire pour voir s'il convient à la bataille; dans le $k a-$ takeleusmos, il invective le serpent, dans l'iambikon il le combat. Cette partie comprend l'iambikon, les coups de salpinx et le grincement de dents, lorsque

41. Strabon, Géographie, IX, 3, 10.

42. Pollux, Dictionnaire (Onomasticon), IV, 84. 


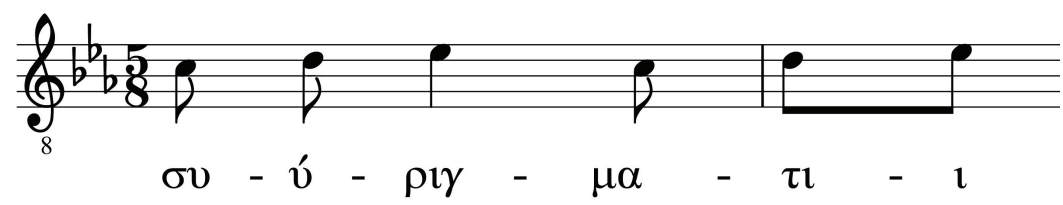

FIG. 1.- Mélodie accompagnant le mot syrigma dans l'hymne d'Athenaios (d'après Bélis 1992).

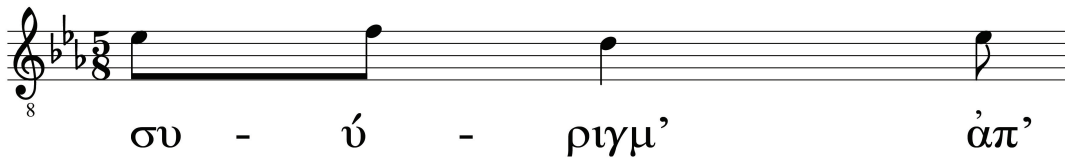

FIg. 2.- Mélodie accompagnant le mot syrigma dans l'hymne de Limenios (d'après Bélis 1992)

le serpent, frappé à mort par les flèches, grince des dents. Le spondeion évoque la victoire du dieu. Dans la katachoreusis, le dieu mène la danse de la victoire. "

C'est le moment où Apollon assène le coup de grâce et où la bête pousse ses derniers sifflements, son dernier râle. Certains ont imaginé que pour imiter ces sifflements, on faisait intervenir la syrinx, au vu du nom que porte cette partie. Cette hypothèse n'a guère de fondement et doit être aussitôt oubliée : un aulète ou un cithariste ne saurait changer d'instrument ou faire appel à un autre musicien ; et on voit mal qu'une flûte de Pan soit particulièrement appropriée pour imiter des sifflements.

Le détour toutefois par la syrinx nous permet de comprendre comment on peut imiter les sifflements par la musique. Nous avons tâché de mettre en évidence précédemment l'ambiguïté qu'il y a dans la conception du sifflement par les Anciens. Si pour nous le sifflement du serpent est plutôt sourd, comme il l'était sans doute aussi pour eux, le mot syrigma permet une plus grande latitude d'interprétation. Ainsi un sifflement sonore peut-il évoquer, ou mieux, rendre un sifflement sourd. L'important pour un Grec est d'entendre un syrigma, plus que d'entendre le sifflement du serpent à proprement parler. Aussi l'aulos est-il particulièrement adapté à faire entendre ce son-là du serpent : instrument à anche, il produit un son nasillard dont la vibration n'est pas très éloignée du sifflement. Quelques coups peuvent donc parfaitement imiter les derniers sifflements de Python ou de Méduse, qui meurt dans le nome polycéphale. Certains ont même pensé que ce nome tirait son nom de la chevelure de serpents qui coiffe le monstre : le nome serait ainsi l'évocation de toutes ces têtes de serpents qui poussent leurs derniers sifflements à la décapitation de Méduse. On peut toutefois avoir une autre interprétation qui vise plutôt le grand nombre de parties du nome, « tête » pouvant être compris comme notre moderne chapitre.

\section{LE DERNIER RÂLE : DU SIFFLEMENT AU THRÈNE}

Pindare explique dans la XIIe Pythique - et c'est développé par la scholie à ce passage - que le nome polycéphale a surtout pour but d'imiter la plainte d'Euryale, sœur de Méduse :

[...] Mís $\alpha$

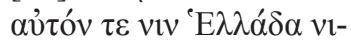

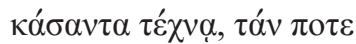

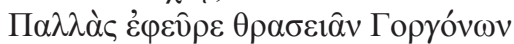

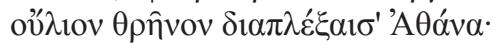

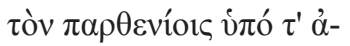

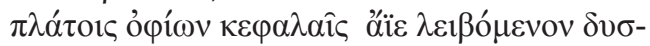

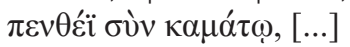




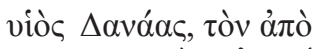

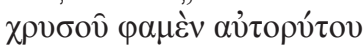

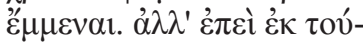

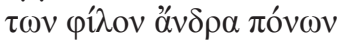

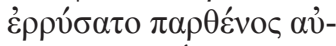

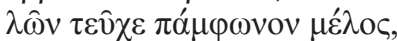

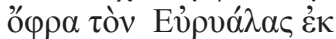

$\kappa \alpha \rho \pi \alpha \lambda \mu \alpha \hat{\alpha} v \gamma \varepsilon v v^{\omega} \omega v$

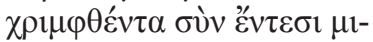

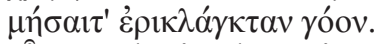

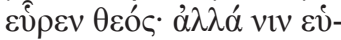

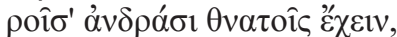

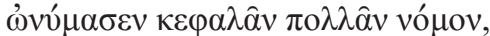

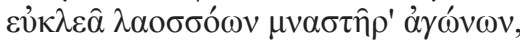

$\lambda \varepsilon \pi \tau o \hat{~ \delta i \alpha v ı \sigma o ́ ~} \mu \varepsilon v o v$

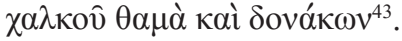

«Midas

lui-même, vainqueur de la Grèce

dans l'art que jadis

Pallas inventa, quand des farouches Gorgones

elle, Athéna, tressa le thrène sinistre,

tel qu'elle l'entendit s'échapper de leurs bouches virginales

et de leurs têtes horribles de serpents,

dans leur douleur poignante, [...]

le fils de Dané, que nous disons

né d'une pluie d'or.

Mais quand elle eut sauvé

de ces labeurs ce héros qui lui était cher,

la déesse vierge forgea une mélodie

riche de tous les sons sur les auloi,

afin d'imiter avec cet équipement

la plainte sonore

d'Euryale,

jaillie de ses joues fébriles.

La déesse l'inventa ; et l'ayant inventé,

elle en fit cadeau aux mortels,

elle l'appela nome aux nombreuses têtes,

air glorieux qui évoque les luttes des peuples,

et que laisse s'écouler le bronze léger

en même temps que les roseaux."

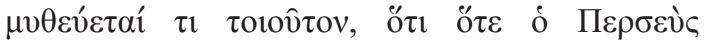

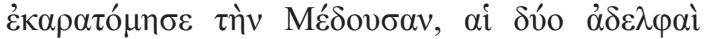

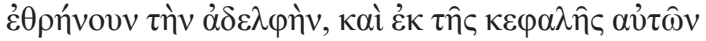

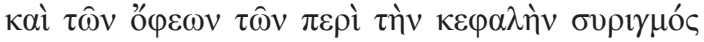

43. Pindare, Pythiques, XII, 10-18, 29-44.

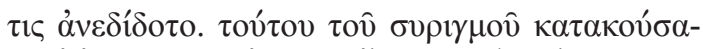

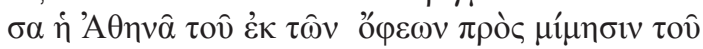

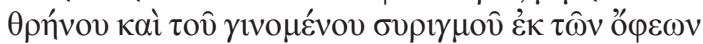

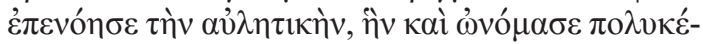

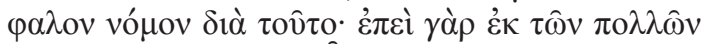

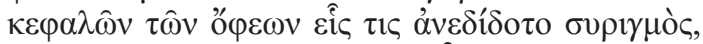

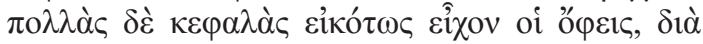

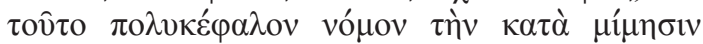

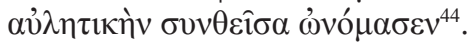

" Il raconte un tel mythe, parce que, lorsque Persée a décapité Méduse, les deux sœurs ont entonné un thrène pour leur sœur, et de leur tête comme des serpents qui entourent leur tête un sifflement s'est élevé. Comme elle avait entendu ce sifflement qui venait des serpents, Athéna créa un air d'aulétique pour imiter le thrène et le sifflement qui était venu des serpents. Cet art, elle l'appela nome polycéphale pour la raison suivante : comme un sifflement était sorti des nombreuses têtes de serpents et que les serpents avaient naturellement de nombreuses têtes, elle l'appela nome polycéphale, ayant composé un air d'aulétique par imitation. »

Cette plainte, goos en grec, désigne un gémissement où l'on devine la déformation de la bouche. Il s'agit donc moins d'un sifflement que d'un gémissement. Mais le nome devait probablement imiter les deux, car Euryale et Sthennô, les sœurs de Méduse, portaient elles aussi une chevelure de serpents sifflants. Nonnos de Panopolis parle de celle de Méduse et de Sthennô dans ses Dionysiaques. C'est donc assurément une plainte sifflante que pousse Euryale :

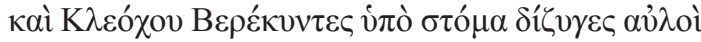

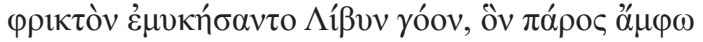

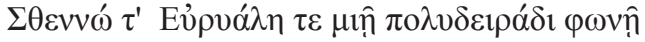

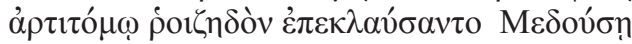

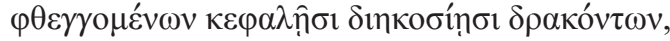

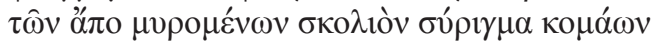

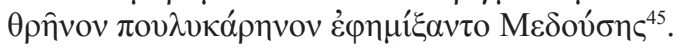

"Les auloi doubles de Bérécynthe, dans la bouche de Kléochos

firent mugir le terrible gémissement libyen, qu'autrefois toutes deux,

44. Scholies aux Pythiques de Pindare, XII, 15b.

45. Nonnos de Panopolis, Dionysiaques, XL, 227-233. 
Sthennô et Euryale, d'une seule voix aux nombreux cous, poussèrent dans leurs pleurs sifflants pour Méduse tout juste décapitée,

quand les serpents criaient de leurs deux cents têtes. C'est pour cela qu'on appela le sifflement tortueux de leurs chevelures qui dégouttaient

thrène aux nombreuses têtes de Méduse. »

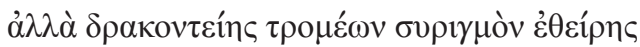

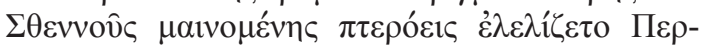
$\sigma \varepsilon v \varsigma^{46}$

«Tremblant devant le sifflement de la chevelure de serpents de Sthennô en furie, Persée ailé s'agitait. "

C'est du combat relaté par Pindare que la déesse garde le trophée qui orne son égide, la tête de la Gorgone. C'est elle qui arme le bras de Persée et fait de lui le vainqueur de la lutte. Si elle invente l'aulos, c'est pour commémorer cet événement : le morceau qu'elle invente est donc un air de victoire, un hymne guerrier. Plus qu'une Athéna guerrière, c'est une Athéna meurtrière dont Pindare fait le portrait et c'est une mise à mort brutale que raconte le nome.

Le hurlement d'Euryale s'accompagne du sifflement des serpents qui constituent la chevelure du monstre. On peut aisément imaginer que même si Pindare ne parle que d'Euryale, le nome visait aussi l'imitation du dernier cri de Méduse. Le son de l'aulos tient donc à la fois d'un hurlement strident et d'un ultime sifflement, comme celui du serpent dans le nome pythique. Le morceau de musique qu'Athéna crée n'est pas qu'un simple chant de victoire : c'est avant tout un thrène, un chant de deuil. Si ce n'est le bras d'Athéna, c'est néanmoins sa volonté qui tue la Gorgone. Or on trouve une légende selon laquelle Athéna tue la Gorgone, car elle voulait se mesurer à elle en beautée ${ }^{47}$.

On peut se demander si le nome pythique visait à imiter seulement les derniers sifflements du serpent ou s'il n'y avait pas un autre

46. Nonnos de Panopolis, Dionysiaques, XXV, 53-54.

47. Pseudo-Apollodore, Bibliothèque, II, 4. élément comme dans le nome polycéphale qui est un véritable "plaidoyer pour l'aulos" (Papadopoulou \& Pirenne-Delforge 2001 : 42). En effet, mettre en scène un monstre permet de prendre certaines libertés. Python est présenté comme un serpent généralement, mais l'iconographie archaïque en fait un être bien plus complexe. Ainsi, un lécythe à figures noires conservé au Musée du Louvre ${ }^{48}$ montre le combat d'Apollon contre Python. Certes, le dessin manque de précision et de netteté, mais il est évident que Python ici n'est pas représenté comme un simple serpent, comme il le sera sur des monnaies notamment. C'est bien plutôt un être anguipède : le bas de son corps est celui d'un serpent mais il a un torse et une tête d'homme, avec un appendice sur le front peu aisé à reconnaître, éventuellement une sorte d'uraeus. Comme beaucoup de monstres de la mythologie, Python peut avoir des attributs anthropomorphes : sans aller jusqu'à lui prêter la voix de la raison, on peut se demander si pour les Anciens, Python pourrait avoir eu une autre voix que le seul sifflement.

C'est toute l'ambiguïté que nous avions soulevée dans la première partie de cette étude. Le sifflement doit-il être considéré comme appartenant à la voix du serpent, ou bien le serpent a-t-il une voix, faible selon Aristote, et un long sifflement ? Si l'on admet que le sifflement est un élément de la voix du serpent ou même qu'il en est indépendant, c'est que le serpent dispose d'une voix, mais cette voix est difficile à entendre, à moins d'y prêter la plus grande attention. Mais s'agissant de monstre, cette voix peut prendre une ampleur plus importante. Dans le cas d'Euryale, elle peut devenir un long gémissement ; peut-être Python poussait-il lui aussi un dernier râle en plus de ses sifflements et de ses grincements de dents. Dans ce cas-là, on imagine assez facilement l'aulos émettre l'imitation d'une longue plainte, comme le hautbois qui imite le canard

48. Lécythe attique à figures noires, Musée du Louvre (Ca 1915, ABV Paralipomena 294), vers 470-460 avant J.-C. 
dans Pierre et le Loup. D'ailleurs l'aulos est un instrument couramment utilisé dans les funérailles grecques pour ses sonorités aiguës, voire stridentes. Il est donc particulièrement adapté aux circonstances que racontent les nomes pythique et polycéphale.

L'évolution technologique de l'aulos à travers le temps, par l'adjonction de systèmes métalliques permettant d'obturer plus ou un moins les trous et par la multiplication des trous, a sans doute permis d'obtenir des effets encore plus spectaculaires lors de l'exécution de ces nomes : il mérite alors l'adjectif que lui prête Pindare, pamphônos. On peut ainsi glisser du parfaitement monstrueux, ces êtres sifflants comme des serpents - Python, Méduse, ou encore Typhon, même si ce dernier n'apparaît pas dans un nome que nous connaissions -, à la limite avec l'humain. Cette voix monstrueuse, qui a encore des accents bestiaux, car elle est peu articulée, est néanmoins déjà dans le sens, celui de la déploration et de la plainte. Le gémissement ultime des monstres tient presque du thrène.

C'est ainsi que Platon dans les Lois voit dans de telles compositions un mélange de bruits saugrenus, issus aussi bien de bêtes, d'hommes que d'instruments. Ce mélange, qui fait la force de la composition musicale aux yeux des mélomanes de l'Antiquité, apparaît comme un immense fatras aux oreilles du philosophe :

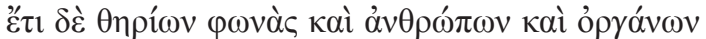

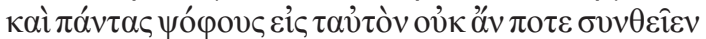

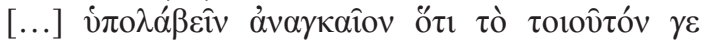

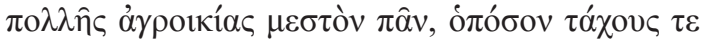

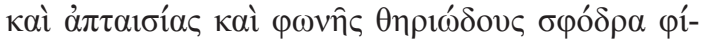

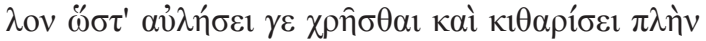

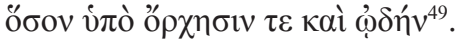

«Les Muses ne sauraient mêler dans la même œuvre des voix de bêtes, d'hommes et d'instruments et toute sorte de sons [...] Il est nécessaire de concevoir qu'un art de cet acabit est plein d'une grande rusticité, tant il aime excessivement la rapidité, la virtuosité et la voix des bêtes, au point d'utiliser le jeu de

49. Platon, Lois, II, 669d et 669e. l'aulos et de la cithare en dehors des circonstances où ils accompagnent la danse et le chant. »

Pour conclure, on pourra dire que le sifflement n'est pas une réalité simple dans le vocabulaire grec. Nous avons tâché de mettre en évidence deux ambiguïtés qu'il porte en lui. La première est son rapport à la voix : il n'est pas facile de saisir avec certitude si le sifflement fait partie de la voix ou s'il est un complément propre aux reptiles, serpents comme tortues. La seconde ambiguïté porte sur le son lui-même, qui oscille entre un son sourd et un bruit plus sonore : les Grecs ne ressentaient apparemment pas le besoin de marquer la différence. Mais de ces ambiguïtés conceptuelles naît une richesse esthétique : l'incertitude qui marque le sifflement représente en même temps un formidable potentiel musical, puisque l'aulos peut parfaitement reproduire toute sonorité sifflotante. Si le hautbois évoque le canard ou le basson le grand-père, c'est l'aulos qui fait penser au serpent. Malheureusement, nous n'avons plus cet instrument et donc il n'y a guère de Pierre et le Serpent en perspective. Et pourtant, la modernité inventera des instruments dont les noms sont liés intimement au serpent, qu'il s'agisse de l'ophicléide ou tout simplement du serpent. Certes ces instruments doivent leur nom à leur forme en $S$ bien plutôt qu'à leur sonorité. Si après Hector Berlioz le serpent est tombé en désuétude, le grand tubiste contemporain $\mathrm{Mi}$ chel Godard l'a remis sur le devant de la scène et s'est amusé à réconcilier instrument et animal dans son album Le Chant du Serpent (1989), où figurent un Reggae des serpents, un Serpent d'or ou encore un Black Snake Blues. Pari osé, si l'on pense à cette réplique de Georg Friedrich Haendel (Van der Straeten 1926 : 609), entendant pour la première fois le son du serpent : "Oh là ! En tout cas, ce n'est pas ce serpent-là qui a séduit Ève!».

\section{Abréviations \\ ABV Paralipomena = BeAzLey J. D. $1971^{2}$.- Paralipo- mena. Additions to Attic black-figure vase-painters and to Attic red-figure vase-painters. Clarendon Press, Oxford.}




\section{BIBLIOGRAPHIE}

Arnott W. G. 1977.- Swan Songs. Greece \& Rome 24 (2) : 149-153.

BÉGuin A. (éd.) 2002 [1964].- E. T. A. Hoffmann, Contes. Fantaisies à la manière de Callot tirées du Journal d'un voyageur enthousiaste 1808-1815. Gallimard, Paris.

BÉLIs A. 1992.- Les hymnes à notation musicale. Corpus des Inscriptions de Delphes, III. De Boccard, Athènes.

BéLIs A. 1999.- Les musiciens dans l'Antiquité. Hachette, Paris.

LABARRIÈrRe J.-L. 1993.- Aristote et la question du langage animal. Métis 8 (1-2) : 247-260.

LABARRIÈrRe J.-L. 2004.- Langage, vie politique et mouvement des animaux. Etudes aristotéliciennes. Vrin, Paris.

Papadopoulou Z. \& Pirenne-Delforge V. 2001.- Inventer et réinventer l'aulos : autour de la XII Pythique de Pindare, in Brulé P. \&
Vendries C. (éds), Chanter les dieux. Musique et religion dans l'Antiquité grecque et romaine. PUR, Rennes : 37-58.

Platel R. [1995] 2005.- Système nerveux et organe des sens, in ВаUснот R. (éd.), Serpents. Artémis, Paris : 50-59.

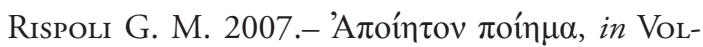
pe Cacciatore P. (ed.), Musica e generi letterari nella Grecia di età classica, Atti del II Congresso Consulta Universitaria Greco (Fisciano, 1 dicembre 2006), Università degli studi di Salerno. Quaderni del Dipartimento di Scienze dell'Antichità 33, Naples : 71-98.

Skoda F. 1983.- La syrinx dans le vocabulaire de l'anatomie en grec ancien, in Froldefond C. (éd.), Mélanges offerts à Édouard Delebecque, Université de Provence ; Marseille Jeanne Laffitte, Aix-en-Provence : 379-391.

Van der Straeten E. 1926.- Handel's Use of Uncommon Instruments and Combinations. The Musical Times 67 : 607-610. 
